\title{
Responses of Thoracic Spinal Interneurons to Vestibular Stimulation
}

\author{
D. M. Miller ${ }^{1}$, D. A. Reighard ${ }^{1}$, A.S. Mehta ${ }^{1}$, A. S. Mehta ${ }^{1}$, R. Kalash ${ }^{2}$, and B. J. Yates ${ }^{1,2}$ \\ 1 Department of Otolaryngology, University of Pittsburgh, Pittsburgh, PA, USA 15213 \\ 2 Department of Neuroscience, University of Pittsburgh, Pittsburgh, PA, USA 15213
}

\begin{abstract}
Vestibular influences on outflow from the spinal cord are largely mediated via spinal interneurons, although few studies have recorded interneuronal activity during labyrinthine stimulation. The present study determined the responses of upper thoracic interneurons of decerebrate cats to electrical stimulation of the vestibular nerve or natural stimulation of otolith organs and the anterior and posterior semicircular canals using rotations in vertical planes. A majority of thoracic interneurons (74/102) responded to vestibular nerve stimulation at median latencies of $6.5 \mathrm{msec}$ (minimum of $\sim 3$ $\mathrm{msec}$ ), suggesting that labyrinthine inputs were relayed to these neurons through trisynaptic and longer pathways. Thoracic interneuronal responses to vertical rotations were similar to those of graviceptors such as otolith organs, and a wide array of tilt directions preferentially activated different cells. Such responses were distinct from those of cells in the cervical and lumbar enlargements, which are mainly elicited by ear-down tilts and are synchronous with stimulus position when low rotational frequencies are delivered, but tend to be in phase with stimulus velocity when high frequencies are employed. The dynamic properties of thoracic interneuronal responses to tilts were instead similar to those of thoracic motoneurons and sympathetic preganglionic neurons. However, the preferred tilt directions of the interneurons were more heterogeneous than thoracic spinal outputs, showing that the outputs do not simply reflect an addition of local interneuronal activity.
\end{abstract}

\section{Keywords}

Otolith organ; vertical semicircular canal; vestbulo-autonomic responses; cardiovascular; respiration; sympathetic nervous system; spinal cord

\section{Introduction}

\begin{abstract}
Since the earliest studies that mapped the efferents of the vestibular nuclei, it was appreciated that most vestibulospinal projections terminate on interneurons and not directly on motoneurons (Nyberg-Hansen 1964; Nyberg-Hansen and Mascitti 1964; Petras 1967; Wilson and Melvill Jones 1979). As such, spinal interneurons are likely involved in integrating descending vestibular signals with segmental and other inputs, and thus play a large role in the process of sensorimotor transformation. However, few studies have considered the responses of spinal interneurons to vestibular stimulation, and most of this work was focused on interneurons in the cervical or lumbar cord that likely regulate limb movements (e.g., Suzuki et al. 1985; Schor et al. 1986). The limb muscles in cats are activated during side-to-side tilts
\end{abstract}

Address Correspondence To: Dr. Bill Yates, University of Pittsburgh, Department of Otolaryngology, Room 519, Eye and Ear Institute, Pittsburgh, PA 15213, Phone: 1-412-647-9614, FAX: 1-412-647-0108, Email: E-mail: byates@ pitt.edu, URL:

http://www.pitt.edu/ byates/yates.html. 
(roll), with extensor muscles contracting during ear-down rotations to produce righting responses (Money and Scott 1962; Roberts 1978; Wilson and Melvill Jones 1979; Wilson et al. 1986). At low frequencies of sinusoidal rotation, the responses of limb muscles are synchronous with stimulus position, but tend to be in phase with stimulus velocity when high rotational frequencies are employed (Wilson et al. 1986). Interneurons in the lower cervical and lumbar spinal cord have analogous response characteristics (Suzuki et al. 1985; Schor et al. 1986), suggesting that a bulk of the signal integration required to generate appropriate reflexes has been accomplished before the motor commands reach limb motoneurons.

Recent studies have also shown that motor and autonomic outputs from the thoracic spinal cord are influenced by vestibular signals. Motoneurons in the thoracic cord that innervate back, abdominal, and intercostal muscles respond to electrical and natural stimulation of labyrinthine afferents (Wilson et al. 1970a; Wilson et al. 1970b; Yates et al. 1993; Rossiter et al. 1996; Brophy et al. 1997). In addition, the firing of sympathetic nervous system efferents arising from the thoracic spinal cord is altered by vestibular stimulation (Uchino et al. 1970; Yates et al. 1993; Yates and Miller 1994; Ray et al. 1997; Kerman et al. 2000; Zakir et al. 2000; Voustianiouk et al. 2006). Unlike the activity of limb motoneurons, motor and sympathetic outflow from the thoracic spinal cord is modulated by fore-aft (pitch) tilt (Yates and Miller 1994; Rossiter et al. 1996; Brophy et al. 1997). Furthermore, the responses are distinct from those of limb muscles in being synchronous with stimulus position when both low- and highfrequency tilts are delivered (Yates and Miller 1994; Rossiter et al. 1996; Brophy et al. 1997). Such effects are needed to stiffen the trunk during changes in body orientation about the transverse axis (Rossiter et al. 1996; Brophy et al. 1997) and to generate the alterations in regional blood flow required during such postural alterations (Yates and Miller 1994).

If integration and processing of vestibular signals in the thoracic spinal cord mainly occurs at the level of interneurons, it is expected that local interneurons and thoracic outputs would have responses to tilts with similar spatial and temporal characteristics. Such a finding, in combination with previous data from lumbar and cervical interneurons (Suzuki et al. 1985; Schor et al. 1986), would provide evidence to support the hypothesis that spinal output neurons (e.g., motoneurons and sympathetic preganglionic neurons) play little integrative role in generating vestibular responses, as upstream neurons have corresponding activity. In contrast, if the responses of thoracic interneurons were distinct from those of motoneurons and sympathetic preganglionic neurons in the same segments, it would be suspected that signal integration by the output neurons is a key step in shaping their responses. The goal of the current study was to distinguish between these possibilities, and to provide a description of the characteristics of the responses of thoracic interneurons to natural and electrical stimulation of labyrinthine afferents.

\section{Methods and materials}

All procedures on animals performed in this study were approved by the University of Pittsburgh's Institutional Animal Care and Use Committee, and conformed to the Guide for the Care and Use of Laboratory Animals (Institute for Laboratory Animal Research, 1996). Data were collected from 19 purpose-bred adult cats obtained from Liberty Research (Waverly, NY, USA). Five of the animals were employed in experiments considering the effects of electrical stimulation of vestibular afferents on the activity of thoracic interneurons, whereas the other 14 were used in studies that employed natural vertical vestibular stimulation produced by rotating the animal's body about the roll (longitudinal) and pitch (transverse) axes using a computer-controlled hydraulic tilt table (Neurokinetics, Pittsburgh, PA, USA). Extracellular recordings of stable negative or biphasic spikes, which presumably originated from cell bodies, were made from single neurons in the T1-T6 spinal segments. Several criteria were employed to identify a cell as a putative interneuron. In most animals, floating antidromic stimulation 
electrodes were placed into the lateral white matter of spinal segments adjacent to the one sampled for single unit activity to determine if a neuron had ascending or descending projections (see Fig. 1B for an example); these electrodes were inserted by hand through the dorsal root entry zone to a depth of $\sim 2 \mathrm{~mm}$. Typically a single electrode was positioned on each side both rostral and caudal to the segment targeted for recording. Any cells with ascending or descending projections were deemed to be interneurons, despite their position in the gray matter. Another criterion employed was location: only cells demonstrated in postmortem histological reconstructions to have been clearly segregated from motoneurons (unless they had ascending or descending projections) were classified as interneurons. This benchmark excluded interneurons in close proximity to the motor pools from our sample, but assured that motoneurons were discounted. A third criterion, firing rate, was employed to ensure that sympathetic preganglionic neurons were removed from the sample. It has been established that sympathetic preganglionic neurons have very low firing frequencies even when sensory pathways that influence the cells are being activated; the modal firing rate of the sympathetic output cells is only approximately 1 spike/s (Gebber and McCall 1976; Barman and Gebber 1984), whereas pre-sympathetic interneurons have much higher discharge frequencies. Our sample of presumed interneurons had mean spontaneous firing rates of $23 \pm 2$ (SEM) spikes/s; the median was 15 spikes/s and the mode was 19 spikes/s. Because the units in our sample had appreciable spontaneous firing rates, they were unlikely to be preganglionic neurons. However, this criterion also potentially excluded interneurons with low firing rates from the data sample, and it is possible that this population of cells had different responses to vestibular stimulation than units with higher spontaneous activity.

Putative interneurons were also tested to determine if their firing was correlated to chest movements related to ventilation. The timing of these chest movements was ascertained from fluctuations in blood pressure recorded from the abdominal aorta, as shown in Fig. 1A.

\section{Surgical procedures}

Each animal was initially anesthetized with isoflurane vaporized in oxygen. Administration of drugs was accomplished through catheters placed in both femoral veins, and blood pressure was monitored using a transducer (Millar Instruments, Houston, TX, USA) inserted through a femoral artery into the abdominal aorta. An infrared lamp and heating pad were used to maintain rectal temperature between $36-38^{\circ} \mathrm{C}$. The animal's head was placed in a stereotaxic frame modified to pitch the head down $30^{\circ}$, in order to vertically align the vertical semicircular canals (Estes et al. 1975). The stereotaxic frame was mounted on a servo-controlled hydraulic tilt table. Hip pins and two vertebral clamps were used to secure the animal's body; the vertebral clamps were placed on the dorsal processes of the vertebrae immediately rostral and caudal to the 3-4 upper thoracic spinal cord segments exposed by laminectomy. A midcollicular decerebration was performed subsequent to ligation of the carotid arteries and aspiration of the cerebral cortex overlying the rostral brainstem. In the five animals used in experiments employing electrical activation of vestibular afferents, an additional surgical procedure described previously (Yates et al. 1993) was done to prepare the vestibular nerves for bipolar electrical stimulation. The tympanic bulla was exposed on both sides using a ventrolateral approach and opened to expose the promontory. The scala vestibuli was exposed and a silversilver chloride ball electrode insulated except at the tip was inserted in the direction of the vestibule. A second electrode was placed 1-2 $\mathrm{mm}$ away in the vicinity of the oval window. The electrodes were covered with warm paraffin wax and held in place using dental cement. An additional laminectomy was performed in these animals to expose the rostral portion of the C1 spinal segment, to provide for recordings of field potentials from descending vestibulospinal projections in the medial longitudinal fasciculus (MLF). 
Anesthesia was stopped after the decerebration and all other surgical procedures were complete at least $1 \mathrm{~h}$ prior to the onset of recording. The animals were then paralyzed using a $10 \mathrm{mg} / \mathrm{kg}$ intravenous injection of gallamine triethiodide (Sigma, St. Louis, MO, USA). Paralysis was maintained using hourly supplemental injections of $5 \mathrm{mg} / \mathrm{kg}$ of gallamine. During paralysis, animals were artificially respired using a positive-pressure ventilator, and end-tidal $\mathrm{CO}_{2}$ was maintained at 3-4\%. We maintained $\mathrm{CO}_{2}$ at low levels to diminish respiratory-related discharges of thoracic neurons, which might have distorted neuronal activity related to vestibular stimulation. If mechanical instability in recording conditions related to respiration was detected, a bilateral pneumothorax was generated.

At the end of the recording session, animals were killed by a $120 \mathrm{mg} / \mathrm{kg}$ intravenous sodium pentobarbital injection, and the exposed thoracic spinal cord segments were removed and fixed in $10 \%$ Formalin.

\section{Data recording procedures}

Extracellular recordings were made from thoracic neurons using epoxy-insulated tungsten microelectrodes with an impedance of 5 or $12 \mathrm{M} \Omega$ (A\&M Systems, Sequim, WA, USA). Systematic tracking was done in $\sim 0.2-\mathrm{mm}$ steps in the medial-lateral direction and $\sim 0.5-\mathrm{mm}$ steps in the anterior-posterior direction to isolate spontaneously-active neurons. In the 14 experiments where natural vestibular stimulation was employed, combined sinusoidal pitch and roll rotations (typically at $7.5^{\circ}-10^{\circ}$ amplitude and $0.2 \mathrm{~Hz}$ frequency) were delivered during tracking to facilitate identifying units whose activity was modulated by whole-body tilts. In the experiments where electrical vestibular stimulation was utilized, every spontaneouslyactive cell encountered was tested for responses to current delivered to labyrinthine afferents.

Activity recorded by the microelectrode was amplified by a factor of 10,000 and filtered with a bandpass of 300-10,000 Hz. The output of the amplifier was fed into a window discriminator for the delineation of spikes from single units. The discriminator output was sampled at 10,000 Hz using a Micro1401 mk 2 data collection system and Spike-2 version 6 software (Cambridge Electronic Design, Cambridge, UK) running on an Intel Macintosh computer with the Microsoft Windows XP operating system and "Boot Camp" program installed. The raw output from the amplifier was also sampled at $10,000 \mathrm{~Hz}$, to permit a post-hoc determination of whether spike shape remained constant throughout the recording period and unit discrimination had been adequate. Consistency of spike shape was ascertained through visual inspection of the waveforms. Two electrolytic lesions were made at predetermined depths in a subset of recording tracks by passing a $20-\mu \mathrm{A}$ negative current for $2 \mathrm{~min}$, so that locations of units could be reconstructed.

When a neuron was encountered, we determined its spontaneous activity in the absence of stimulation by sampling firing over a period of 30-60 sec. Recordings of blood pressure were made simultaneously (sampled at $100 \mathrm{~Hz}$ using the Cambridge Electronic Design data collection system) to permit a determination of whether the unit's activity was synchronized to oscillations in blood pressure related to artificial ventilation (see Fig. 1A). Subsequently, we tested whether the unit could be antidromically activated by stimulation of spinal segments adjacent to the recording site, by delivering stimulus pulses that were $0.2 \mathrm{~ms}$ in duration and typically had maximal amplitudes of 200-500 $\mu \mathrm{A}$. The presence of antidromic responses to spinal stimulation was confirmed using collision (see Fig. 1B for an example). Following this initial analysis, the unit's responses to electrical or natural stimulation of vestibular afferents were determined as described below. 


\section{Recording and analysis of responses to electrical vestibular stimulation}

In five animals, square-wave current pulses, $0.2 \mathrm{~ms}$ in duration, were employed to stimulate the vestibular nerves. During the implantation of electrodes in the inner ear, the effectiveness of vestibular nerve stimulation was determined by monitoring eye movements related to the vestibulo-ocular reflex (elicited by stimulation using a 50-shock train with a 3-msec interpulse interval), and the difference in intensity required to produce eye and facial movements was noted. The facial movements were due to stimulus spread to motor axons in the facial nerve, the closest non-target nerve to the stimulating electrodes (Gacek 1969; Wilson et al. 1977). During the recording session, the maximal stimulus intensity was limited to values lower than those shown to produce facial movements. Current intensities required to alter the activity of thoracic interneurons were expressed as multiples of the threshold for producing field potentials recorded from the MLF in rostral C1.

To determine whether a putative thoracic interneuron received vestibular inputs, a five-shock train with an interpulse interval of $3 \mathrm{~ms}$ at an intensity five times that required to produce MLF field potentials was employed to activate vestibular afferents. Stimulus trains were delivered at a rate of one per 1-2 s. Shorter trains were used to determine the minimal number of shocks required to produce a response. Response latencies were calculated from the last shock of the shortest train that produced a response, referred to as the 'effective shock'. Additionally, the minimum stimulus intensity required to produce a response when using a five-shock train was determined.

\section{Recording and analysis of responses to natural vestibular stimulation}

In 14 animals, we recorded the responses of putative thoracic interneurons to stimulation of vertical semicircular canals and otolith organs, which was produced by tilting the animal about the pitch and roll axes. Our procedures for performing vertical vestibular simulation have been described in detail previously (Jian et al. 2002). We first determined the plane of tilt that produced maximal modulation of the unit's firing rate (response vector orientation). Response vector orientation was calculated from responses to the "wobble" stimulus, a constantamplitude tilt whose direction moves around the animal at constant speed (Schor et al. 1984). The direction of the response vector orientation lies midway between the maximal response directions to clockwise $(\mathrm{CW})$ and counterclockwise $(\mathrm{CCW})$ wobble stimulation, because the phase differences between stimulus and response are reversed during the two directions of rotation (Schor et al. 1984). Thus, by consideration of both responses, these phase differences could be accounted for. Wobble stimulation was typically delivered at $0.2 \mathrm{~Hz}$, and sometimes at lower and higher frequencies as well. Subsequently, the response vector orientation was confirmed by comparing the gain of responses to tilts in a variety of fixed vertical planes, typically delivered at $0.2 \mathrm{~Hz}$ and at an amplitude of $7.5^{\circ}$. These tilts always included the roll and pitch planes. After a unit's response vector orientation was established, planar tilts at or near this orientation were used to study the dynamics of the vestibular response (i.e., response gain and phase across stimulus frequencies). Response dynamics were routinely determined over the frequency range of $0.1-1 \mathrm{~Hz}$; for some cells rotations at $0.02,0.05 \mathrm{~Hz}$, and/or $2 \mathrm{~Hz}$ were also delivered. The amplitudes of these stimuli were usually $2.5-7.5^{\circ}$ at frequencies $\geq 0.5$ $\mathrm{Hz}, 5-10^{\circ}$ at $0.2 \mathrm{~Hz}$, and $7.5-15^{\circ}$ at lower frequencies.

Neural activity recorded during rotations in vertical planes was binned (1,000 bins/cycle) and averaged over the sinusoidal stimulus period. When a unit's spontaneous activity was rhythmic (e.g., synchronized with ventilation), sufficient sweeps were collected to average-out the oscillations in firing that were unrelated to the stimulus. Sine waves were fitted to responses with the use of a least-squares minimization technique (Schor et al. 1984); an initial analysis was performed using the Spike-2 software as data collection was occurring, and a post-hoc final analysis was subsequently executed using Matlab (The MathWorks, Natick, MA, USA). 
The response sinusoid was characterized by two parameters: phase shift from the stimulus sinusoid (subsequently referred to as phase) and amplitude relative to the stimulus sinusoid (subsequently referred to as gain). Responses were considered significant if the signal-to-noise ratio (see (Schor et al. 1984) for method of calculation) was $\geq 0.5$ and only the first harmonic was prominent.

\section{Reconstruction of recording sites and statistical analysis of data}

The thoracic spinal segments containing recording sites were cut into $50 \mu \mathrm{m}$ transverse sections that were collected serially and stained using thionine. Recording sites were reconstructed using camera lucida relative to electrolytic lesion placement, positions of electrode tracks, and microelectrode depth. The locations of the motor pools were noted in camera lucida drawings, and units were considered to be thoracic interneurons only if they were segregated from motoneurons or could be antidromically activated by stimulation in an adjacent cord segment.

Statistical analyses were performed using Prism 5 software (GraphPad Software, San Diego, CA, USA). Pooled data are presented as means \pm one standard error.

\section{Results}

\section{Responses of thoracic interneurons to electrical stimulation of vestibular afferents}

A mean current intensity of $52 \pm 9 \mu \mathrm{A}$ (median of $45 \mu \mathrm{A}$ ) delivered to the vestibular nerve was needed to elicit field potentials recordable from the MLF, whereas an intensity of $435 \pm 44 \mu \mathrm{A}$ (median of $405 \mu \mathrm{A}$ ) was required to produce stimulus spread to the facial nerve. As such, the maximal currents used to test whether thoracic interneurons received labyrinthine inputs (5 times the MLF field threshold) were well below those that activated fibers in the closest nontarget nerve to the stimulation site.

A total of 102 putative thoracic interneurons were examined for responses to vestibular nerve stimulation: 35 were located in the dorsal horn (Rexed's laminae I-V), 64 were positioned in the intermediate zone (Rexed's laminae VI-VIII and X), and 3 were isolated in the vicinity of motoneurons but could be antidromically activated by stimulation of the white matter caudal to the recording site. Out of this population, 21/35 dorsal horn neurons, 50/64 intermediate zone cells, and all three putative interneurons located in the motor pool responded to activation of labyrinthine afferents. Of the 74 units that received vestibular inputs, the firing of 62 was modulated by stimulation of both labyrinths, 9 were affected only by contralateral stimulation, and 3 responded only to ipsilateral stimulation. Typically, the responses to stimulation of both sides were similar. The largest proportion of cells that received vestibular inputs (39/74) were predominantly excited, 24 were initially excited and then inhibited, 4 were inhibited, and 7 displayed a response consisting of initial inhibition followed by excitation. Examples of responses to vestibular nerve stimulation are shown in Fig. 2, panels A and B.

Fig. 2C shows for each of the units the latency of the earliest response elicited by stimulation of either the ipsilateral or contralateral labyrinth. The latency was calculated from the effective shock, or last shock of the shortest train that evoked changes in neuronal activity; four cells were excluded because they were lost before the effective shock was determined. The majority of cells that received vestibular inputs (51/70) responded to a single shock delivered to the labyrinth, whereas 10 cells required two shocks, 7 needed three shocks, while longer trains were necessary for the others. The response latency for approximately half of the neurons $(32 / 70)$ was $<5.5 \mathrm{msec}$ from the effective shock; these cells were deemed to have short-latency responses. The locations of these neurons in the spinal gray matter, as well as the positions of neurons with medium-latency $(<10.5 \mathrm{msec})$ and long-latency $(\geq 10.5 \mathrm{msec})$ responses to 
vestibular stimulation are shown in Fig. 3B. The mean response latency was $9.4 \pm 1.3 \mathrm{msec}$ from the effective shock, although the median response latency was much shorter: $6.5 \mathrm{msec}$.

A small fraction of the units (10/102) had activity correlated with blood pressure fluctuations related to ventilation. The firing of 7 of these cells was affected by vestibular nerve stimulation; their relative response latencies are reflected in Fig. 3B. In addition, 12 neurons could be antidromically activated by stimulation in an adjacent cord segment; 2 of these cells were located in the dorsal horn, 7 in the intermediate zone, and 3 in the motor pools. Most (9/12) had caudally-projecting axons, although 3 projected rostrally; the mean antidromic threshold for the neurons was $140 \pm 40 \mu \mathrm{A}$, and the mean conduction velocity was $22 \pm 3 \mathrm{~m} / \mathrm{sec}$. The firing of a large majority of the neurons (11/12) with ascending or descending projections was altered by electrical stimulation of the vestibular nerve; the mean response latency was $10 \pm 2 \mathrm{msec}$.

\section{Responses of thoracic interneurons to natural stimulation of vestibular afferents}

We characterized the responses of 66 putative thoracic interneurons to rotations in vertical planes. The locations of these neurons are shown in Fig. 3A; 12 were positioned in the dorsal horn, 53 in the intermediate zone, and 1 cell found in the motor pool was deemed to be an interneuron because it could be antidromically activated by stimulation of the white matter of a caudal spinal segment. Examples of the responses of two neurons to vertical rotations are shown in columns A and B of Fig. 4. Panels 1 and 2 illustrate neuronal activity elicited by wobble rotations, whereas panels 3-5 show responses to different frequencies of roll stimuli.

Bode plots illustrating the dynamic properties of responses of thoracic interneurons to rotations in fixed planes near the response vector orientation are shown in Fig. 5. Both response gain and phase were plotted with respect to stimulus position, such that a response whose phase leads stimulus position by $90^{\circ}$ is synchronous with stimulus velocity. The large majority of neurons $(48 / 66)$ had response phases that remained within $45^{\circ}$ of stimulus position at all frequencies tested (see Fig. 5A). Most of these cells (35/48) also had response gains that remained relatively consistent across stimulus frequencies, increasing $<3$-fold per stimulus decade. Only about $10 \%(5 / 48)$ of the thoracic interneurons with response phases synchronous with stimulus position had response gains that increased $>7$-fold per stimulus decade. As such, the changes in firing of a majority of thoracic interneurons during vertical vestibular stimulation suggested that they received inputs from graviceptors. However, a few units had alternate response dynamics. Three cells had response phases near stimulus velocity $\left(90^{\circ}\right)$ at all frequencies tested, and seven neurons had responses that were synchronous with stimulus position when low rotational frequencies were employed that advanced to near stimulus velocity when high-frequency tilts were delivered. The response dynamics of these units are illustrated in Fig. 5B. The responses of nine cells developed a phase lag as stimulus frequency increased: four lagged the velocity of the tilt $\left(90^{\circ}\right)$ whereas five lagged stimulus position $\left(0^{\circ}\right)$, as indicated in Fig. 5C. The response gains of these cells remained relatively consistent across stimulus frequencies, increasing no more than 5 -fold per stimulus decade.

The response vector orientations of thoracic interneurons are shown in Fig. 6. Fig. 6A depicts data for cells whose response phases were near stimulus position at all frequencies of wholebody rotation. The directions of tilt that optimally activated these neurons were highly varied, with 18 being nearest (within $22.5^{\circ}$ ) to a canal plane, 14 being nearest to pitch, and 16 being nearest to roll. Although a few cells activated by roll tilts had particularly large responses to rotations, the median response gains to wobble stimuli were similar for units that were maximally affected by rotations near the roll, pitch, and canal planes. For example, the median response gain to the highest-frequency wobble stimulus delivered was $1.2 \mathrm{spikes} / \mathrm{s} /{ }^{\circ}$ for cells best activated by roll-plane stimuli, $1.1 \mathrm{spikes} / \mathrm{s} /{ }^{\circ}$ for neurons that responded best to pitch, and $1.0 \mathrm{spikes} / \mathrm{s} /{ }^{\circ}$ for units maximally affected by canal-plane rotations. In addition, most (36/48) of the graviceptive neurons were excited by ipsilateral ear down rotations, whereas 12 were 
excited by contralateral ear down tilts. Neurons with other than graviceptive responses to vertical vestibular stimulation similarly had a wide diversity of response vector orientations, but were more commonly excited by contralateral than ipsilateral tilts (see Fig. 6B-C). For the large majority of cells (60/66), the gains of responses to the two directions ( $\mathrm{CW}$ and $\mathrm{CCW}$ ) of wobble rotation varied no more than $2: 1$. Neurons that receive vestibular inputs with different spatial and temporal properties, and thus can be classified as having spatiotemporal convergence (STC) behavior, typically have responses to $\mathrm{CW}$ and CCW wobble rotations of greatly different magnitude (at least $>2: 1$ ) because the misaligned inputs to the cell add during one direction of rotation but subtract when rotations are delivered in the opposite direction (Wilson et al. 1986; Kasper et al. 1988; Schor and Angelaki 1992). As such, it appears that few thoracic interneurons have STC responses to rotations in vertical planes.

Less than a quarter of the neurons that responded to natural vestibular stimulation (15/66) had activity that was synchronized with ventilation; in addition, putative thoracic interneurons included in our sample did not exhibit spontaneous oscillations in activity other than those related to breathing. The responses of this subset of units did not appear to differ from the population as a whole. For example, the response vector orientations of these cells were just as varied as those for neurons that lacked ventilator-related activity: 6 were best activated by rotations near the plane of a semicircular canal, 6 responded best to pitch oscillations, and 3 were best stimulated by roll tilts. In addition, 6 of the thoracic interneurons that responded to vertical vestibular stimulation could be antidromically activated by stimulation of the white matter of an adjacent spinal segment. All 6 of the antidromically-activated units were located in the intermediate zone or motor pool; 4 had rostrally-projecting axons and two projected caudally. Their conduction velocities ranged from 10-30 m/sec (median of $18 \mathrm{~m} / \mathrm{sec}$ ), and the antidromic thresholds ranged from 50-360 $\mu \mathrm{A}$ (median of $185 \mu \mathrm{A}$ ). All of the interneurons with ascending or descending projections had response vector orientations near the plane of a semicircular canal, and most (4/6) had dynamic responses to tilts similar to graviceptors.

\section{Discussion}

The present findings suggest that a large fraction of thoracic interneurons receive labyrinthine inputs, and that about half of these cells respond to vestibular nerve stimulation at latencies < $5.5 \mathrm{msec}$. Prior studies have shown that vestibular nucleus neurons that receive monosynaptic inputs from VIII ${ }^{\text {th }}$ nerve afferents respond to stimulation of the labyrinth at latencies $<1.4$ msec (Zhang et al. 2001; Kushiro et al. 2008), whereas an average of 0.6-0.7 msec is required to elicit monosynaptic EPSPs in upper thoracic neurons following stimulation of descending projections at the level of the brainstem (Wilson et al. 1970a; Wilson et al. 1970b). Even considering that an additional $\sim 0.5 \mathrm{msec}$ would be needed for the excited neurons to generate an action potential, only cells with response latencies $<3 \mathrm{msec}$ from the effective shock could safely be classified as receiving disynaptic inputs from the inner ear. As such, although many thoracic interneurons receive relatively short-latency vestibular signals, these are mainly relayed through trisynaptic and longer pathways.

The spatial and temporal properties of responses of thoracic interneurons to vertical vestibular stimulation were distinct from those of interneurons located in the $\mathrm{C} 7$ segment of the cervical enlargement, which were established using a hydraulic tilt table very similar to the one employed in the present study (Schor et al. 1986). The majority of cervical enlargement interneurons (15/26) were classified as having "velocity responses," or phases that were near stimulus velocity when rotational frequencies $\geq 0.2 \mathrm{~Hz}$ were delivered (a combination of the "phase near $90^{\circ}$ " and "advancing phase" categories utilized in the present study)(Schor et al. 1986), whereas only $9 / 66$ cells in the upper thoracic spinal cord had such response properties. As such, velocity responses are less prevalent amongst thoracic interneurons than C7 interneurons ( $\mathrm{p}<0.001$, Fisher's exact test). Furthermore, $69 \%$ of cervical enlargement 
interneurons had response vector orientations near the roll plane and the remaining $31 \%$ were best activated by rotations near a canal plane (Schor et al. 1986). In contrast, only $33 \%$ of upper thoracic interneurons were best activated by roll rotations, whereas $41 \%$ and $26 \%$ of the cells had response vector orientations nearest a canal plane or the pitch axis, respectively. A $\chi^{2}$ analysis revealed that the distribution of response vector orientations differed between the two populations of cells $(\mathrm{p}<0.001)$. Neurons in the lumbar cord have response dynamics that are much more similar to neurons in the cervical cord than to thoracic interneurons. For example, out of 46 neurons studied in the L3-L4 segments in decerebrate cats, 39 had response vector orientations closer to the roll than to the pitch axis (Suzuki et al. 1985); this distribution of response vector orientations is significantly different than for thoracic neurons $(\mathrm{p}<0.001$, Fisher's exact test). It thus appears that the vestibular signals sent to the upper thoracic spinal cord are distinct from those relayed to segments that control limb movements; in particular, the thoracic cord appears to receive fewer inputs related to stimulus velocity, but more signals reflecting changes in body orientation around the transverse axis.

The activity of a majority of cervical interneurons during vertical vestibular stimulation is similar to that of forelimb muscles; both are stimulated by tilts about the longitudinal axis and have graviceptive responses to low rotational frequencies but velocity responses at high frequencies (Wilson et al. 1986). In contrast, muscles innervated by thoracic motoneurons whose activities have been examined during vertical vestibular stimulation as well as thoracic sympathetic outflow are activated by tilts about the transverse axis and have graviceptive responses across the spectrum of frequencies tested (Yates and Miller 1994; Rossiter et al. 1996; Brophy et al. 1997). As such, the dynamic properties of responses of most thoracic interneurons to rotations in vertical planes were similar to those of thoracic output. However, the matching of the spatial properties of the responses was not precise. In particular, the response vector orientations of approximately half of the upper thoracic interneurons (34/66) were closer to the roll plane than the pitch plane. The simple addition of signals from these interneurons would not generate a response aligned with the transverse axis as is observed in thoracic output neurons (Yates and Miller 1994; Rossiter et al. 1996; Brophy et al. 1997). It thus appears that the responses of thoracic sympathetic preganglionic neurons and motoneurons to vestibular stimulation are not a simple reflection of the activity already manifested in the firing of local interneurons, and that many output neurons perform an integration of signals with spatial properties distinct from those manifested in their discharges. Perhaps the signal processing required by thoracic motoneurons and sympathetic neurons is more extensive than that done by forelimb and hindlimb motoneurons.

A limitation of this study is that we could not ascertain the targets of the interneurons whose activity was recorded. Prior studies have shown that respiratory interneurons in the thoracic spinal cord are located in the intermediate zone dorsomedial to the motoneurons, and outnumber the motoneurons by a 10:1 ratio (Kirkwood et al. 1988; Kirkwood et al. 1993; Schmid et al. 1993). Our sample included neurons in this area, although only a few displayed rhythmic discharges related to respiration (it would be presumed that the respiratory rhythm in these experiments was coupled with mechanical ventilation, particularly since large air volumes were employed to maintain end-tidal $\mathrm{CO}_{2} \leq 4 \%$ in most animals (Petrillo and Glass 1984)). However, the low $\mathrm{CO}_{2}$ also likely diminished the magnitude of the central respiratory rhythm, such that respiratory interneurons were difficult to recognize. Presympathetic interneurons have been identified in the vicinity of the intermediolateral cell column, the dorsal horn, and the intermediate zone (Barman and Gebber 1984; Tang et al. 2003; Tang et al. 2004; Schramm 2006); vestibular-activated cells were located in all of these regions. The contribution of interneurons to regulating back muscle activity has not been ascertained, but presumably such premotor neurons are present in the thoracic spinal cord. However, the fact that vestibular inputs to thoracic interneurons were so prevalent (e.g., 74/102 cells responded 
to electrical stimulation of the vestibular nerve) suggests that all of these subgroups of units, and likely others, receive inputs from the inner ear.

In summary, the present data show that vestibular inputs to upper thoracic spinal interneurons are prevalent, and distinct from those to other cord segments where neuronal responses to vertical vestibular stimulation have been examined. In particular, cells in the thoracic cord appear to receive fewer inputs related to stimulus velocity, but more signals reflecting changes in body orientation around the transverse axis, than neurons in the cervical and lumbar enlargements. As such, the dynamic properties of responses of thoracic interneurons to vertical rotations are more closely aligned than those of other spinal neurons to the responses of thoracic motoneurons and sympathetic preganglionic neurons, which have graviceptive responses to rotations in the pitch plane. Nonetheless, the response vector orientations of the thoracic interneurons were heterogeneous, with only half being better activated by pitch than roll rotations. As such, the activity of the thoracic output cells, which is modulated by pitch rotations, does not reflect a simple addition of the diverse responses of the local interneurons to vestibular stimulation. Rather, a more complex integrative process must occur at the level of the output cells.

\section{Acknowledgements}

The authors thank Lucy Cotter, Allison Waggoner, and Michael Bonadio for technical assistance. Funding was provided by Grant R01-DC03732 from the National Institutes of Health (USA). Core support was provided by NIH grant P30-DC05205.

\section{References}

Barman SM, Gebber GL. Spinal interneurons with sympathetic nerve-related activity. Am J Physiol 1984;247:R761-767. [PubMed: 6093601]

Brophy GM, Rossiter CD, Bolton PS, Yates BJ. Vestibular influences on cat lumbar paravertebral muscles. Neurosci Lett 1997;223:189-192. [PubMed: 9080464]

Estes MS, Blanks RH, Markham CH. Physiologic characteristics of vestibular first-order canal neurons in the cat. I. Response plane determination and resting discharge characteristics. J Neurophysiol 1975;38:1232-1249. [PubMed: 1177015]

Gacek RR. The course and central termination of first order neurons supplying vestibular end organs in the cat. Acta-Otolaryngol Suppl 1969;254:1-66. [PubMed: 4914344]

Gebber GL, McCall RB. Identification and discharge patterns of spinal sympathetic interneurons. Am J Physiol 1976;231:722-733. [PubMed: 184713]

Institute for Laboratory Animal Research. Guide for the Care and Use of Laboratory Animals. National Academy Press; Washington, D.C.: 1996.

Jian BJ, Shintani T, Emanuel BA, Yates BJ. Convergence of limb, visceral, and vertical semicircular canal or otolith inputs onto vestibular nucleus neurons. Exp Brain Res 2002;144:247-257. [PubMed: 12012162]

Kasper J, Schor RH, Wilson VJ. Response of vestibular neurons to head rotations in vertical planes. I. Response to vestibular stimulation. J Neurophysiol 1988;60:1753-1764. [PubMed: 3199179]

Kerman IA, McAllen RM, Yates BJ. Patterning of sympathetic nerve activity in response to vestibular stimulation. Brain Res Bul 2000;53:11-16.

Kirkwood PA, Munson JB, Sears TA, Westgaard RH. Respiratory interneurones in the thoracic spinal cord of the cat. J Physiol 1988;395:161-192. [PubMed: 3411478]

Kirkwood PA, Schmid K, Sears TA. Functional identities of thoracic respiratory interneurones in the cat. J Physiol 1993;461:667-687. [PubMed: 8350279]

Kushiro K, Bai R, Kitajima N, Sugita-Kitajima A, Uchino Y. Properties and axonal trajectories of posterior semicircular canal nerve-activated vestibulospinal neurons. Exp Brain Res 2008;191:257264. [PubMed: 18830591] 
Money KE, Scott JW. Functions of separate sensory receptors of nonauditory labyrinth of the cat. Am J Physiol 1962;202:1211-1220. [PubMed: 14475366]

Nyberg-Hansen R. Origin and termination of fibers from the vestibular nuclei descending in the medial longitudinal fasciculus. An experimental study with silver impregnation methods in the cat. J Comp Neurol 1964;122:355-367. [PubMed: 14184859]

Nyberg-Hansen R, Mascitti TA. Sites and mode of termination of fibers of the vestibulospinal tract in the cat. An experimental study with silver impregnation methods. J Comp Neurol 1964;122:369383. [PubMed: 14184860]

Petras JM. Cortical, tectal and tegmental fiber connections in the spinal cord of the cat. Brain Res 1967;6:275-324. [PubMed: 6060511]

Petrillo GA, Glass L. A theory for phase locking of respiration in cats to a mechanical ventilator. Am J Physiol 1984;246:R311-320. [PubMed: 6584038]

Ray CA, Hume KM, Shortt TL. Skin sympathetic outflow during head-down neck flexion in humans. Am J Physiol Regul Integr Comp Physiol 1997;273:R1142-R1146.

Roberts, TDM. Neurophysiology of Postural Mechanisms. Butterworths; London: 1978.

Rossiter CD, Hayden NL, Stocker SD, Yates BJ. Changes in outflow to respiratory pump muscles produced by natural vestibular stimulation. J Neurophysiol 1996;76:3274-3284. [PubMed: 8930272]

Schmid K, Kirkwood PA, Munson JB, Shen E, Sears TA. Contralateral projections of thoracic respiratory interneurones in the cat. J Physiol 1993;461:647-665. [PubMed: 8350278]

Schor RH, Angelaki DE. The algebra of neural response vectors. Annals of the New York Academy of Sciences 1992;656:190-204. [PubMed: 1599143]

Schor RH, Miller AD, Tomko DL. Responses to head tilt in cat central vestibular neurons. I. Direction of maximum sensitivity. J Neurophysiol 1984;51:136-146. [PubMed: 6319622]

Schor RH, Suzuki I, Timerick SJB, Wilson VJ. Responses of interneurons in the cat cervical cord to vestibular tilt stimulation. J Neurophysiol 1986;56:1147-1156. [PubMed: 3491186]

Schramm LP. Spinal sympathetic interneurons: their identification and roles after spinal cord injury. Prog Brain Res 2006;152:27-37. [PubMed: 16198691]

Suzuki I, Timerick SJB, Wilson VJ. Body position with respect to head or body position in space is coded by lumbar interneurons. J Neurophysiol 1985;54:123-133. [PubMed: 3875695]

Tang X, Neckel ND, Schramm LP. Locations and morphologies of sympathetically correlated neurons in the T(10) spinal segment of the rat. Brain Res 2003;976:185-193. [PubMed: 12763252]

Tang X, Neckel ND, Schramm LP. Spinal interneurons infected by renal injection of pseudorabies virus in the rat. Brain Res 2004;1004:1-7. [PubMed: 15033414]

Uchino Y, Kudo N, Tsuda K, Iwamura Y. Vestibular inhibition of sympathetic nerve activities. Brain Res 1970;22:195-206. [PubMed: 5458666]

Voustianiouk A, Kaufmann H, Diedrich A, Raphan T, Biaggioni I, Macdougall H, Ogorodnikov D, Cohen B. Electrical activation of the human vestibulo-sympathetic reflex. Exp Brain Res 2006;171:251261. [PubMed: 16308690]

Wilson VJ, Gacek RR, Maeda M, Uchino Y. Saccular and utricular input to cat neck motoneurons. J Neurophysiol 1977;40:63-73. [PubMed: 833629]

Wilson, VJ.; Melvill, Jones G. Mammalian Vestibular Physiology. Plenum Press; New York: 1979.

Wilson VJ, Schor RH, Suzuki I, Park BR. Spatial organization of neck and vestibular reflexes acting on the forelimbs of the decerebrate cat. J Neurophysiol 1986;55:514-526. [PubMed: 3485706]

Wilson VJ, Yoshida M, Schor RH. Monosynaptic excitation and inhibition of thoracic motoneurons by fibers in the medial longitudinal fasciculus. Brain Res 1970a;18:181-184. [PubMed: 4313891]

Wilson VJ, Yoshida M, Schor RH. Supraspinal monosynaptic excitation and inhibition of thoracic back motoneurons. Exp Brain Res 1970b;11:282-295. [PubMed: 4248931]

Yates BJ, Jakus J, Miller AD. Vestibular effects on respiratory outflow in the decerebrate cat. Brain Res 1993;629:209-217. [PubMed: 8111625]

Yates BJ, Miller AD. Properties of sympathetic reflexes elicited by natural vestibular stimulation: implications for cardiovascular control. J Neurophysiol 1994;71:2087-2092. [PubMed: 7931504]

Zakir M, Ono S, Meng H, Uchino Y. Saccular and utricular influences on sympathetic nerve activities in cats. Exp Brain Res 2000;134:402-406. [PubMed: 11045365] 
Zhang X, Zakir M, Meng H, Sato H, Uchino Y. Convergence of the horizontal semicircular canal and otolith afferents on cat single vestibular neurons. Exp Brain Res 2001;140:1-11. [PubMed: 11500792] 

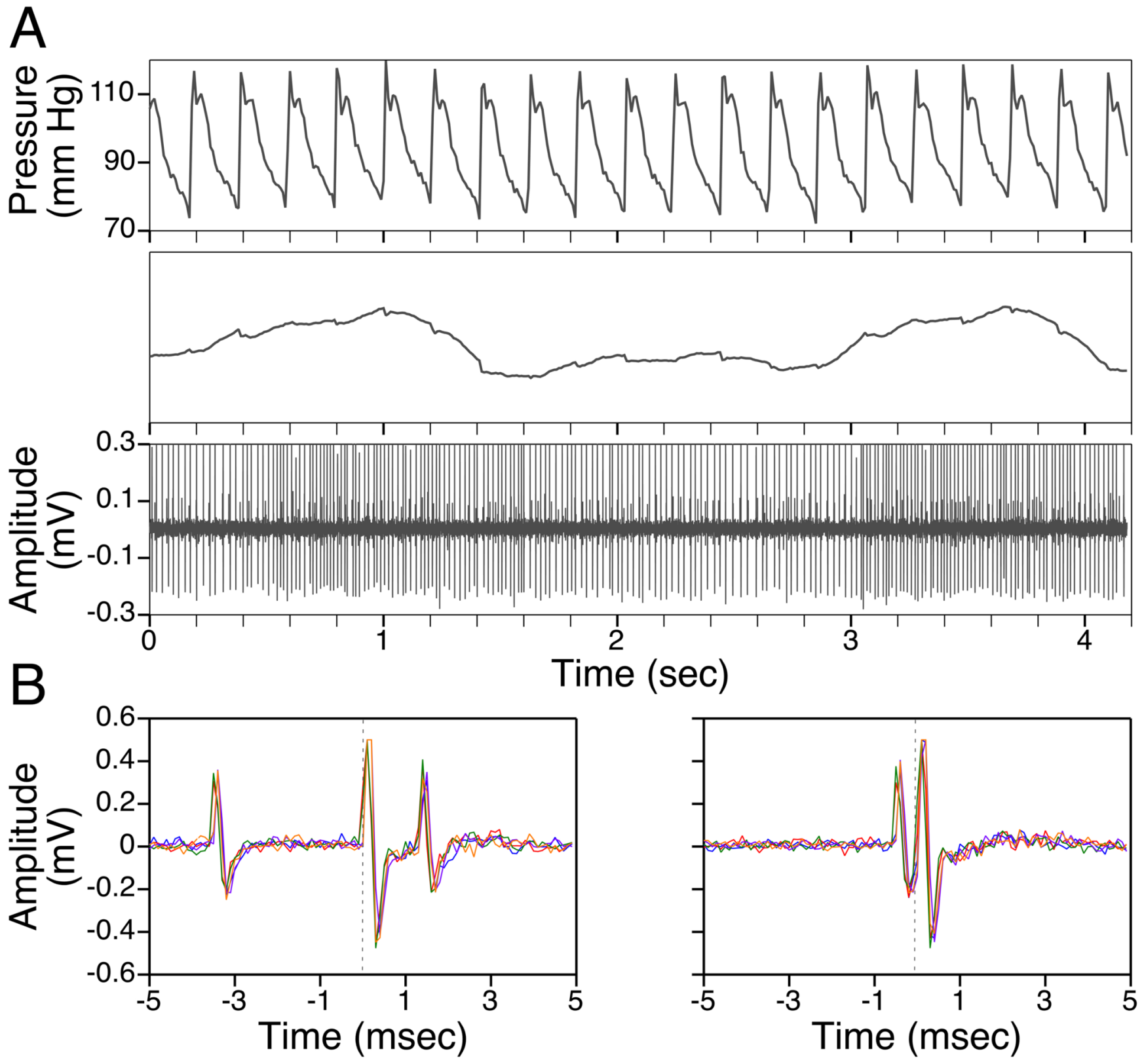

Figure 1.

A: Comparison of the firing of a neuron (bottom trace) with fluctuations in blood pressure (top panels). The uppermost panel depicts a raw blood pressure recording, whereas the middle panel shows the same data after smoothing using a $0.2 \mathrm{~s}$ time constant. Slow alterations in blood pressure at the frequency of ventilation are evident; the unit's firing oscillated in phase with these respiration-related blood pressure fluctuations. B: Antidromic activation of a putative thoracic interneuron from the contralateral white matter caudal to the recording site. The $70 \mu \mathrm{A}$ stimulus was delivered at time zero, and five overlapping sweeps are shown. In the left panel, an antidromic response is evident when the triggering spike preceded the stimulus by $3.5 \mathrm{msec}$. However, when the interval between triggering spikes and the stimulus was reduced below $1 \mathrm{msec}$ (right panel), collision occurred. 

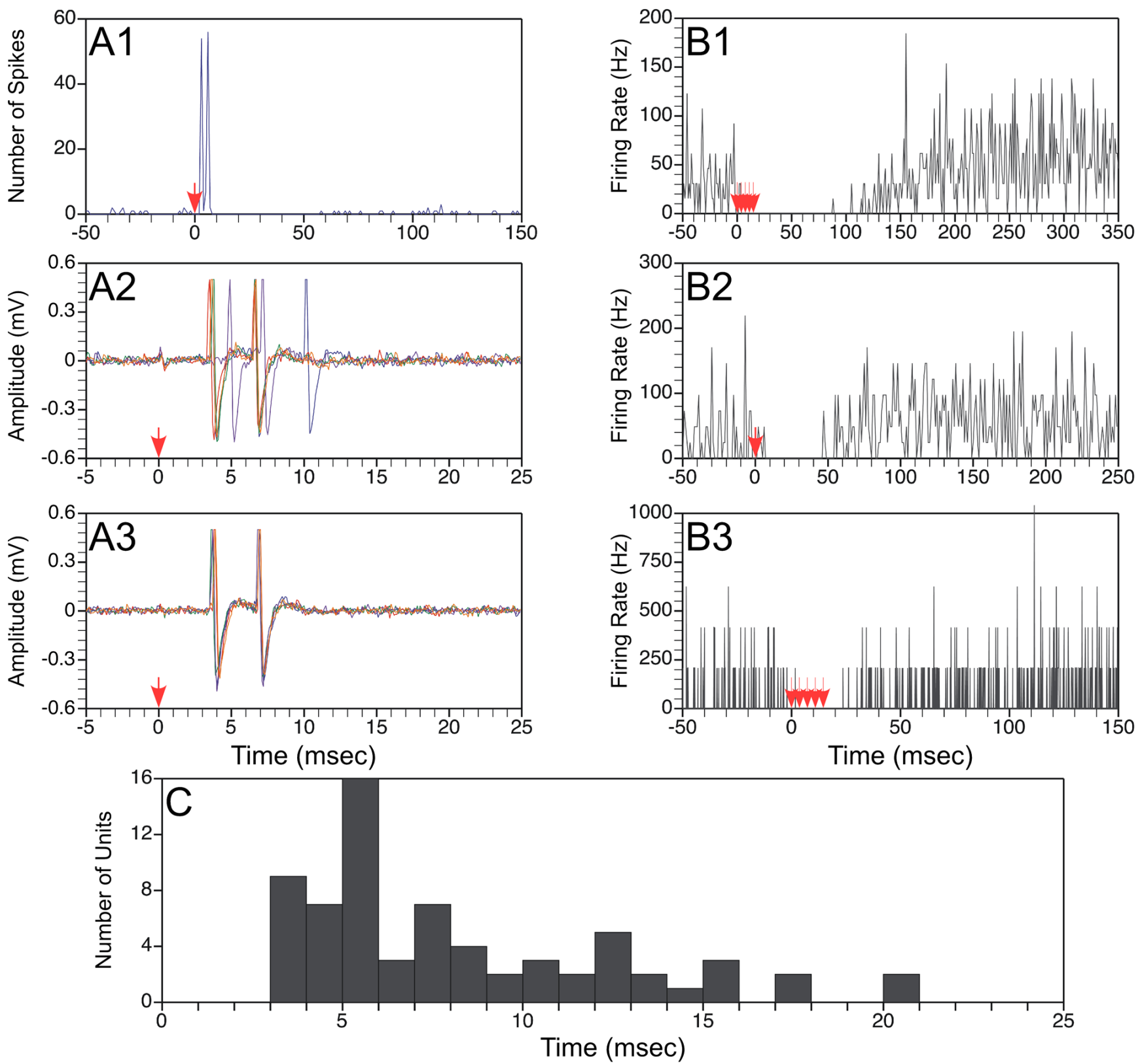

Figure 2.

Responses of thoracic neurons to electrical stimulation of the vestibular nerve. A: Responses of a neuron that exhibited short-latency excitation followed by inhibition during vestibular stimulation. Trace A1 is a poststimulus histogram showing the response to application of a single shock at $2 \mathrm{~T}(80 \mu \mathrm{A})$ intensity to the ipsilateral labyrinth; the shock was delivered at time zero, as designated by an arrow. The histogram was generated from data collected following 55 stimulus repetitions. Traces $\mathbf{A} 2$ and $\mathbf{A} 3$ respectively show raw unit activity in response to a single shock delivered at $5 \mathrm{~T}(200 \mu \mathrm{A})$ or $2 \mathrm{~T}(80 \mu \mathrm{A})$ intensity; 5 sweeps are superimposed in each panel. B: Poststimulus histograms illustrating the responses of a neuron that was inhibited by stimulation of the contralateral labyrinth. Trace B1 shows the response to a train of 5 shocks at $5 \mathrm{~T}(200 \mu \mathrm{A})$ intensity; B2 illustrates the effects of a single shock at 5T, whereas B3 indicates the effects of 5 shocks delivered at $2 \mathrm{~T}(80 \mu \mathrm{A})$. C: the latency of the earliest 
response, elicited by stimulation of either the ipsilateral or contralateral labyrinth, of all units. Response latencies were measured from the effective shock. Data were excluded for two units whose response latencies were $>25 \mathrm{msec}$. 


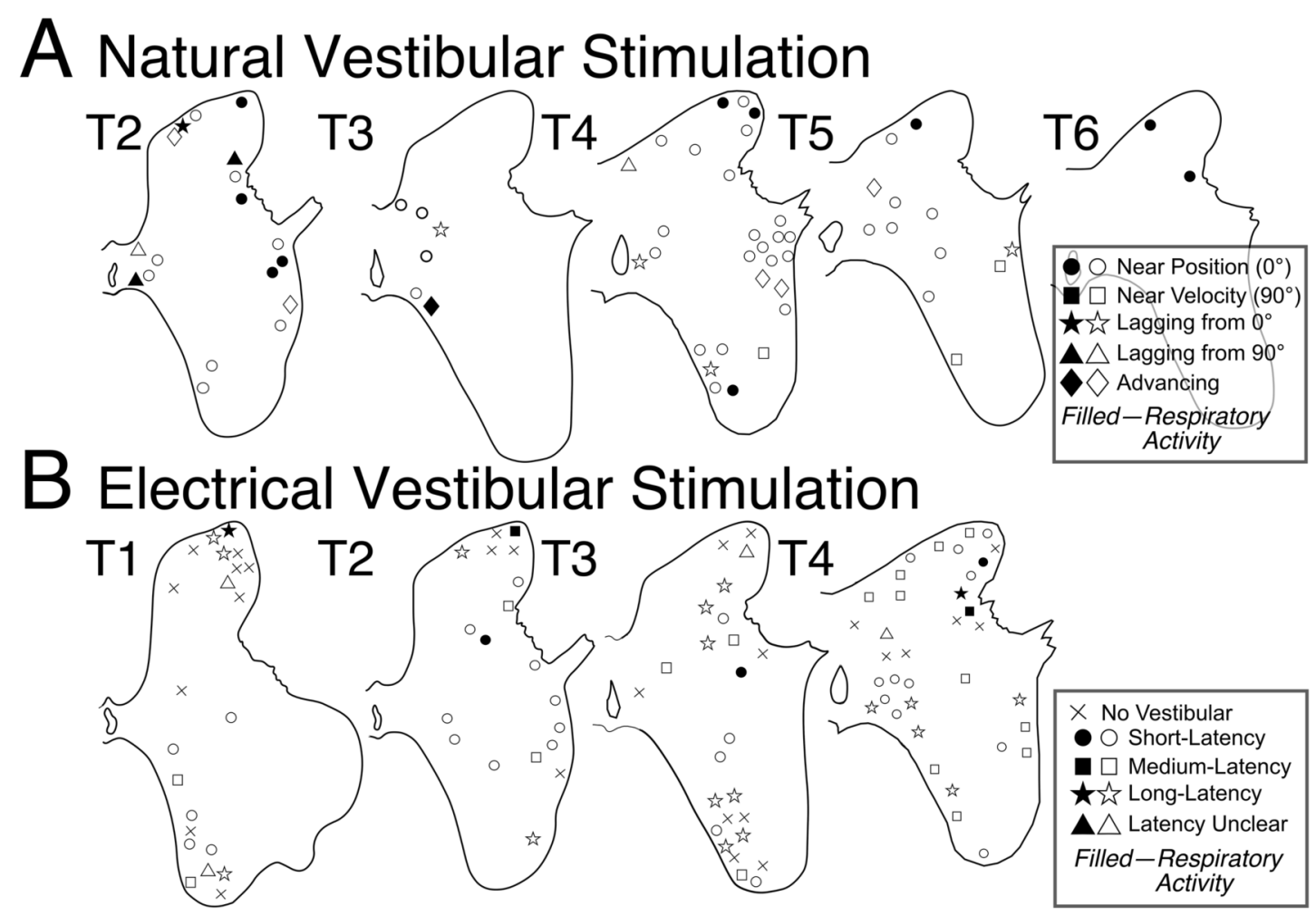

Figure 3.

Locations of putative interneurons plotted on transverse sections of the upper thoracic spinal gray matter; the segment depicted in each diagram is indicated. A: Locations of neurons whose responses to natural vestibular stimulation were characterized. Symbols of different types are used to designate positions of units whose response phases were within $45^{\circ}$ of stimulus position at all rotational frequencies (near position), were within $45^{\circ}$ of stimulus velocity at all frequencies (near velocity), developed a phase lag with respect to stimulus position (lagging from $0^{\circ}$ ) or velocity (lagging from $90^{\circ}$ ) as rotational frequency was increased, or exhibited a response phase near stimulus position at low rotational frequencies that advanced to near stimulus velocity at high frequencies (advancing). Shaded symbols indicate neurons with firing related to ventilation. B: Locations of neurons tested for responses to electrical stimulation of the vestibular nerve. Symbols of different types are used to designate whether a unit failed to respond to vestibular nerve stimulation (no vestibular), or whether the cell's firing was altered at a latency $<5.5 \mathrm{msec}$ (short-latency), 5-5-10.5 msec (medium-latency), or $>10.5 \mathrm{msec}$ (long-latency). Cells that responded to vestibular stimulation but whose response latency was not established are designated as "latency unclear." 

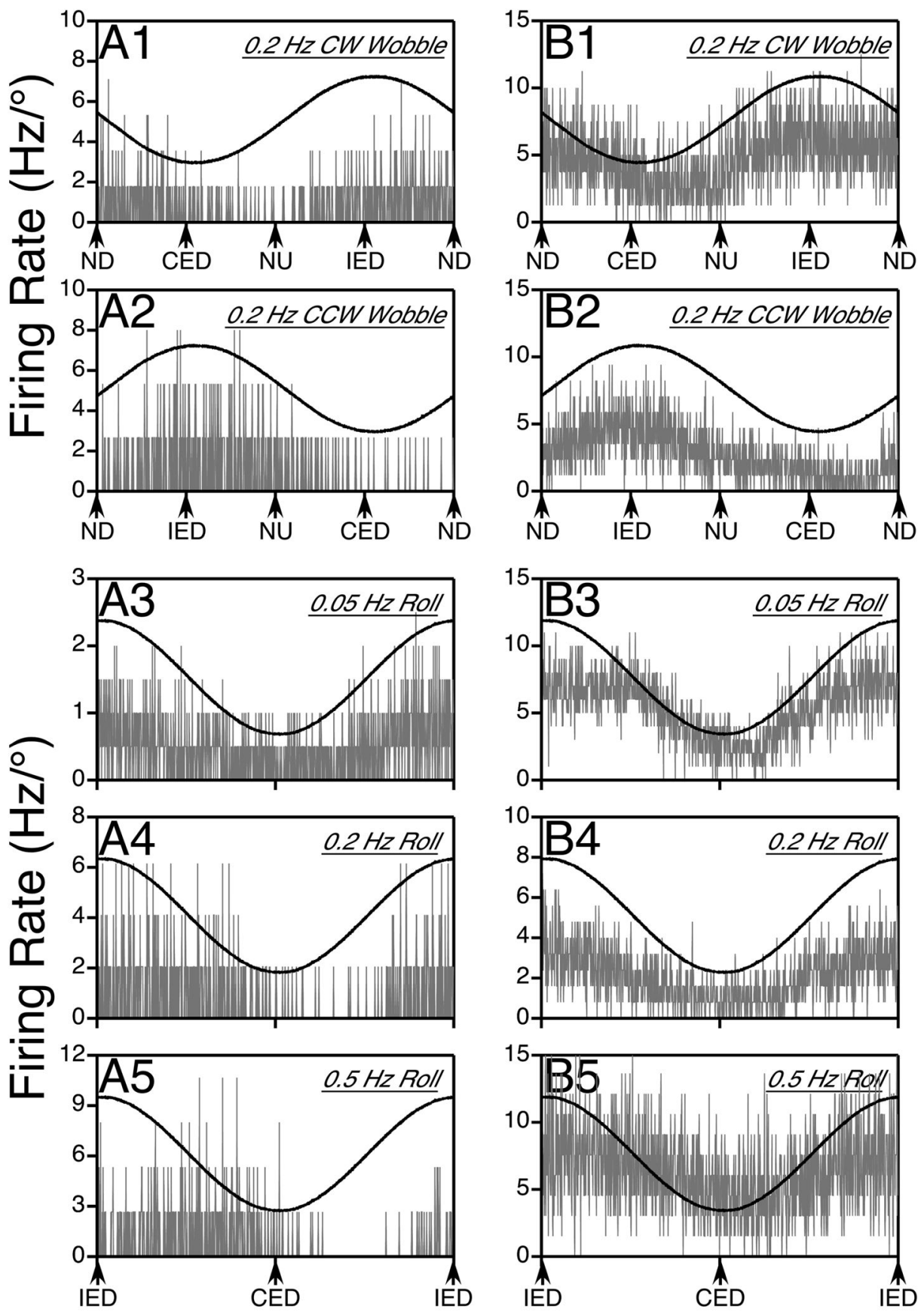

Figure 4.

Responses of two putative thoracic interneurons to sinusoidal rotations in vertical planes; gray traces show average neuronal firing rate (in $\mathrm{Hz}$ per degree of rotation), whereas black traces indicate the position of the tilt table. Column $\mathbf{A}$ illustrates data for a neuron whose responses lagged stimulus position as rotational frequency was increased, whereas column $\mathbf{B}$ provides data for a cell with responses in phase with tilt position. In each column, traces $\mathbf{1}$ and $\mathbf{2}$ respectively show the responses to $0.2 \mathrm{~Hz} \mathrm{CW}$ and $\mathrm{CCW}$ wobble stimuli, whereas traces 3-5 depict the responses to $0.05 \mathrm{~Hz}$ (trace 3), $0.2 \mathrm{~Hz}$ (trace 4), and $0.5 \mathrm{~Hz}$ (trace 5 ) roll rotations. The responses illustrated in panels $\mathbf{A 1}, \mathbf{A 2}, \mathbf{A 4}$, and $\mathbf{A 5}$ were elicited by $7.5^{\circ}$ rotations, whereas the others were produced by $10^{\circ}$ tilts. Approximately 15 sweeps were averaged when wobble 
stimuli were delivered (traces A1, A2, B1, B2), whereas the following number of sweeps were averaged during roll tilts: 9 for trace $\mathbf{A 3}, 13$ for $\mathbf{A 4}, 24$ for $\mathbf{A 5}, 5$ for $\mathbf{B 3}, 20$ for $\mathbf{B 4}$, and 33 for B5. Abbreviations: $C E D$, contralateral ear-down roll; $\mathrm{CW}$, clockwise; $\mathrm{CCW}$, counterclockwise; IED, ipsilateral ear-down roll, $N D$, nose-down pitch, $N U$, nose-up pitch. 

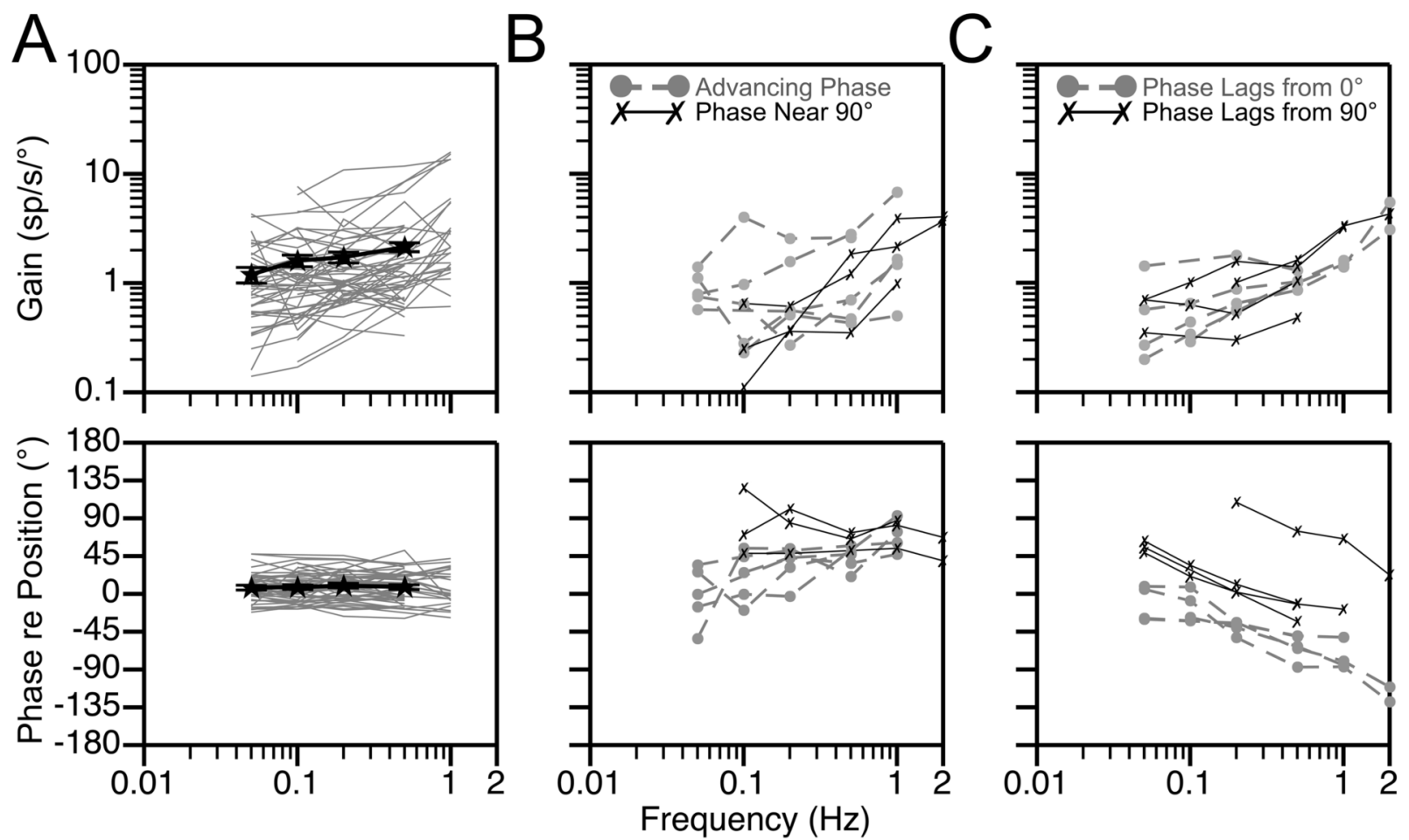

Figure 5.

Bode plots illustrating the dynamic properties of responses of putative thoracic interneurons to rotations at multiple frequencies. Response gain and phase are plotted relative to stimulus position. A: Bode plots for neurons whose response phases remained near stimulus position at all frequencies. Thin gray lines designate responses of individual neurons, whereas thick black lines show averaged data for all units. Error bars designate one standard error; for some points the errors were so small that the bars are not obvious. B: Bode plots for neurons whose response phases remained within $45^{\circ}$ of stimulus velocity at all frequencies (phase near $90^{\circ}$ ) or exhibited a response phase near stimulus position at low rotational frequencies that advanced to near stimulus velocity at high frequencies (advancing phase). C: Bode plots for neurons whose responses developed a phase lag with respect to stimulus position (phase lags from $0^{\circ}$ ) or velocity (phase lags from $90^{\circ}$ ) as rotational frequency was increased. No data averages are provided in panels $\mathrm{B}$ or $\mathrm{C}$, because so few cells exhibited the response characteristics that are highlighted. 


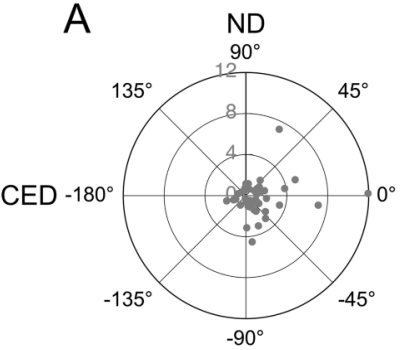

NU

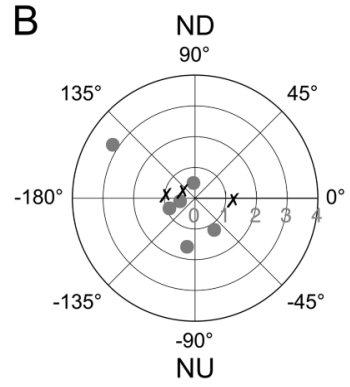

- Advancing Phase

$x$ Phase Near $90^{\circ}$

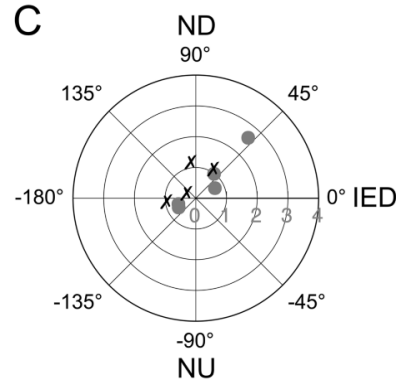

- Phase Lags from $0^{\circ}$

$x$ Phase Lags from $90^{\circ}$

Figure 6.

Polar plots showing the response vector orientations and gains for thoracic interneurons, determined using wobble stimuli that were usually delivered at $0.2 \mathrm{~Hz}$. Numbers along the radius of each plot indicate gain $\left(\right.$ spikes $/ \mathrm{s}^{\circ}{ }^{\circ}$ ). A: Data for neurons whose response phases remained near stimulus position at all frequencies. B: Data for neurons whose response phases remained within $45^{\circ}$ of stimulus velocity at all frequencies (phase near $90^{\circ}$ ) or exhibited a response phase near stimulus position at low rotational frequencies that advanced to near stimulus velocity at high frequencies (advancing phase). C: Data for neurons whose responses developed a phase lag with respect to stimulus position (phase lags from $0^{\circ}$ ) or velocity (phase lags from $90^{\circ}$ ) as rotational frequency was increased. Abbreviations: $C E D$, contralateral eardown roll; $I E D$, ipsilateral ear-down roll; $N D$, nose-down pitch; $N U$, nose-up pitch. 